\title{
Crystal structure of 2,6,6-trimethyl-2-hydroxybicyclo[3.1.1]-3-heptanone oxime, $\mathrm{C}_{10} \mathrm{H}_{17} \mathrm{NO}_{2}$
}

\author{
L.-X. Zhang*, M.-L. Hu, A.-J. Zhang and G.-Q. Xiang \\ Wenzhou Normal College, Department of Chemistry and Material Science, Wenzhou, 325027, P. R. China
}

Received May 13, 2002, accepted and available on-line August 26, 2002; CCDC-No. 1267/866

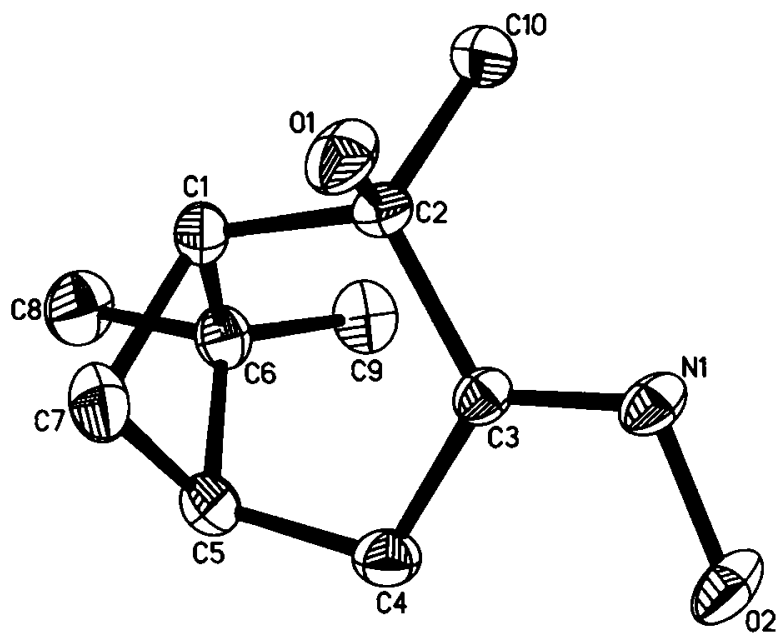

Abstract

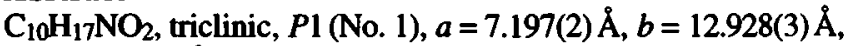
$c=17.966(4) \AA, \alpha=87.14(1)^{\circ}, \beta=81.75(2)^{\circ}, \gamma=79.78(2)^{\circ}$,

$V=1627.5 \AA^{3}, Z=6, R_{\mathrm{gt}}(F)=0.035, w R_{\text {ref }}\left(F^{2}\right)=0.077$,

$T=298 \mathrm{~K}$.

\section{Source of material}

The title compound was synthesized through the oxidization of $\alpha$-pinene by potassium permanganate in $90 \%$ aqueous acetone at $273 \mathrm{~K}-283 \mathrm{~K}$ and then the reaction of intermediate 2,6,6-trimethyl-2-hydroxybicyclo[3.1.1]-3-heptanone with hydroxylamine hydrochloride. The single crystals were grown by recrystallization from ethyl acetate-petroleum.

\section{Discussion}

As a part of a work on the ligand behaviour and reactivity of aminoalcohols, here we report the characterization of a precursor of aminoalcohols, 2,6,6-trimethyl-2-hydroxybicyclo[3.1.1]3-heptanone oxime.

The distance $\mathrm{C} 3-\mathrm{N} 1$ is equal to 1.270 (3) $\AA$, clearly indicating a double bond. The bond lengths of $\mathrm{C} 1-\mathrm{C} 2, \mathrm{C} 1-\mathrm{C} 6, \mathrm{C} 2-\mathrm{C} 3$, $\mathrm{C} 4-\mathrm{C} 5, \mathrm{C} 5-\mathrm{C} 6, \mathrm{C} 6-\mathrm{C} 8, \mathrm{C} 1-\mathrm{C} 7, \mathrm{C} 2-\mathrm{C} 10, \mathrm{C} 3-\mathrm{C} 4$, $\mathrm{C} 5-\mathrm{C} 7, \mathrm{C} 6-\mathrm{C} 9$ are $1.515(4) \AA, 1.570(4) \AA, 1.523(4) \AA$, $1.512(4) \AA, 1.536(4) \AA, 1.526(4) \AA, 1.535(4) \AA, 1.511(4) \AA$, $1.514(4) \AA, 1.536(4) \AA, 1.508(4) \AA$, respectively; these data suggest that single bonds exist between every two carbon atoms and the $\mathrm{C} 2=\mathrm{C} 3$ double bond have disappeared [1]. The distance $\mathrm{O} 1-\mathrm{C} 2$ is equal to $1.451(3) \AA$, which verify the appearance of hydroxy group $[2,3]$. The distance $\mathrm{O} 2-\mathrm{N} 1$ is equal to $1.403(3) \AA$, which confirms the existence of another hydroxy group. The atoms $\mathrm{C} 1, \mathrm{C} 5, \mathrm{C} 6$ and $\mathrm{C} 7$ constitute a four-membered ring. The bond an-

\footnotetext{
* Correspondence author (e-mail: hml64@sohu.com)
}

gles $\angle \mathrm{Cl}-\mathrm{C} 7-\mathrm{C} 5, \angle \mathrm{C} 7-\mathrm{C} 1-\mathrm{C} 6, \angle \mathrm{C} 7-\mathrm{C} 5-\mathrm{C} 6$ and $\angle \mathrm{C} 5-\mathrm{C} 6-\mathrm{Cl}$ are $86.2(2)^{\circ}, 87.3(2)^{\circ}, 88.5(2)^{\circ}$ and $85.0(2)^{\circ}$, respectively, and other $\mathrm{C}-\mathrm{C}-\mathrm{C}$ bond angle is about $109^{\circ}$. The bond angle $\angle \mathrm{C} 3-\mathrm{N} 1-\mathrm{O} 2$ is $111.5^{\circ}$. Every unit cell is composed of six molecules, there are twelve intermolecular hydrogen bonds in a unit cell, the hydrogen bond length is between $2.6 \AA$ to $2.9 \AA$, and the hydrogen bond angle is in the range of $149^{\circ}-173^{\circ}$. The structural differences among the six independent molecules are not significant.

Table 1. Data collection and handling.

$\begin{array}{ll}\text { Crystal: } & \text { colorless plate, size } 0.20 \times 0.52 \times 0.80 \mathrm{~mm} \\ \text { Wavelength: } & \text { Mo } K_{\alpha} \text { radiation }(0.71073 \AA) \\ \mu: & 0.77 \mathrm{~cm}^{-1} \\ \text { Diffractometer, scan mode: } & \text { Siemens P4, } \omega \\ 2 \theta_{\text {max: }} & 50^{\circ} \\ N(h k l)_{\text {measured, }} N(h k l)_{\text {unique }}: & 6444,6240 \\ \text { Criterion for } I_{\text {obs }}, N(h k l)_{\text {gl }}: & I_{\text {obs }}>2 \sigma\left(I_{\text {obs }}\right), 4274 \\ N(\text { param })_{\text {refined: }} & 734 \\ \text { Programs: } & \text { SHELXTL-plus [4], SHELXL-93 [5], } \\ & \text { ORTEP3 [6] }\end{array}$

Table 2. Atomic coordinates and displacement parameters (in $\AA^{2}$ ).

\begin{tabular}{|c|c|c|c|c|c|}
\hline Atom & Site & $x$ & $y$ & $z$ & $U_{\text {iso }}$ \\
\hline$H(10)$ & $1 a$ & 0.4029 & -0.2323 & 1.4908 & 0.072 \\
\hline $\mathrm{H}(2 \mathrm{O})$ & $1 a$ & 0.3495 & -0.0994 & 1.2694 & 0.080 \\
\hline$H(110)$ & $1 a$ & 0.4544 & -0.4323 & 1.5934 & 0.075 \\
\hline $\mathrm{H}(12 \mathrm{O})$ & $1 a$ & 0.4286 & -0.2917 & 1.3468 & 0.099 \\
\hline $\mathrm{H}(210)$ & $1 a$ & 0.4238 & -0.5225 & 1.6888 & 0.080 \\
\hline $\mathrm{H}(22 \mathrm{O})$ & $1 a$ & 0.5072 & -0.2097 & 1.6205 & 0.094 \\
\hline$H(310)$ & $1 a$ & 0.2338 & -0.0661 & 1.1690 & 0.093 \\
\hline $\mathrm{H}(32 \mathrm{O})$ & $1 a$ & 0.2225 & -0.0886 & 0.9328 & 0.113 \\
\hline $\mathrm{H}(410)$ & $1 a$ & 0.2152 & 0.0355 & 1.0707 & 0.078 \\
\hline $\mathrm{H}(42 \mathrm{O})$ & $1 a$ & 0.2669 & 0.3040 & 0.8948 & 0.107 \\
\hline $\mathrm{H}(510)$ & $1 a$ & 0.2666 & 0.0992 & 0.9153 & 0.089 \\
\hline $\mathrm{H}(520)$ & $1 a$ & 0.3551 & 0.3337 & 0.7388 & 0.092 \\
\hline $\mathrm{H}(1)$ & $1 a$ & 0.7559 & -0.1340 & 1.5553 & 0.060 \\
\hline $\mathrm{H}(4 \mathrm{~A})$ & $1 a$ & 0.6276 & 0.0339 & 1.3448 & 0.069 \\
\hline $\mathrm{H}(4 \mathrm{~B})$ & $1 a$ & 0.4305 & 0.0588 & 1.3963 & 0.069 \\
\hline $\mathrm{H}(5)$ & $1 a$ & 0.6685 & 0.1237 & 1.4549 & 0.071 \\
\hline $\mathrm{H}(7 \mathrm{~A})$ & $1 a$ & 0.5874 & 0.0402 & 1.5744 & 0.080 \\
\hline $\mathrm{H}(7 \mathrm{~B})$ & $1 a$ & 0.4324 & 0.0008 & 1.5320 & 0.080 \\
\hline $\mathrm{H}(8 \mathrm{~A})$ & $1 a$ & 1.0524 & -0.0481 & 1.5310 & 0.102 \\
\hline $\mathrm{H}(8 \mathrm{~B})$ & $1 a$ & 0.8880 & 0.0455 & 1.5558 & 0.102 \\
\hline $\mathrm{H}(8 \mathrm{C})$ & $1 a$ & 1.0378 & 0.0586 & 1.4850 & 0.102 \\
\hline $\mathrm{H}(9 \mathrm{~A})$ & $1 a$ & 1.0176 & -0.0130 & 1.3661 & 0.090 \\
\hline $\mathrm{H}(9 \mathrm{~B})$ & $1 a$ & 0.8923 & -0.0996 & 1.3620 & 0.090 \\
\hline $\mathrm{H}(9 \mathrm{C})$ & $1 a$ & 1.0682 & -0.1228 & 1.4063 & 0.090 \\
\hline$H(10 A)$ & $1 a$ & 0.7770 & -0.3065 & 1.4867 & 0.077 \\
\hline $\mathrm{H}(10 \mathrm{~B})$ & $1 a$ & 0.8671 & -0.2486 & 1.4155 & 0.077 \\
\hline
\end{tabular}


Table 2. Continued.

\begin{tabular}{|c|c|c|c|c|c|}
\hline Atom & Site & $x$ & $y$ & $z$ & $U_{\text {iso }}$ \\
\hline $\mathrm{H}(10 \mathrm{C})$ & $1 a$ & 0.6926 & -0.3041 & 1.4108 & 0.077 \\
\hline $\mathrm{H}(11)$ & $1 a$ & 0.1863 & -0.6095 & 1.5996 & 0.062 \\
\hline $\mathrm{H}(\mathrm{J} 4 \mathrm{~A})$ & $\mathrm{l} a$ & 0.5035 & -0.5610 & 1.3788 & 0.084 \\
\hline$H(14 B)$ & $1 a$ & 0.2971 & -0.5241 & 1.3579 & 0.084 \\
\hline$H(15)$ & $1 a$ & 0.3216 & -0.7041 & 1.4040 & 0.077 \\
\hline $\mathrm{H}(17 \mathrm{~A})$ & $1 a$ & 0.5234 & -0.6487 & 1.5074 & 0.081 \\
\hline $\mathrm{H}(\mathrm{J} / \mathrm{B})$ & la & 0.4019 & -0.7412 & 1.5282 & 0.081 \\
\hline$H(18 A)$ & $1 a$ & -0.0756 & -0.6976 & 1.5520 & 0.099 \\
\hline $\mathrm{H}(18 \mathrm{~B})$ & $1 a$ & 0.1218 & -0.7691 & 1.5278 & 0.099 \\
\hline $\mathrm{H}(18 \mathrm{C})$ & $1 a$ & -0.0230 & -0.7385 & 1.4696 & 0.099 \\
\hline$H(19 A)$ & $1 a$ & -0.0808 & -0.5637 & 1.4151 & 0.090 \\
\hline H(19B) & $1 a$ & 0.0135 & -0.4735 & 1.4407 & 0.090 \\
\hline $\mathrm{H}(19 \mathrm{C})$ & $1 a$ & -0.1445 & -0.5182 & 1.4958 & 0.090 \\
\hline$H(11 A)$ & $1 a$ & 0.1202 & -0.4090 & 1.6302 & 0.075 \\
\hline$H(11 B)$ & $1 a$ & 0.0118 & -0.3992 & 1.5597 & 0.075 \\
\hline $\mathrm{H}(11 \mathrm{C})$ & $1 a$ & 0.1654 & -0.3286 & 1.5648 & 0.075 \\
\hline $\mathrm{H}(21)$ & $1 a$ & 0.6483 & -0.5920 & 1.8429 & 0.067 \\
\hline$H(24 A)$ & $1 a$ & 0.3995 & -0.2644 & 1.8088 & 0.075 \\
\hline$H(24 B)$ & $1 a$ & 0.6154 & -0.2535 & 1.7949 & 0.075 \\
\hline $\mathrm{H}(25)$ & $1 a$ & 0.5412 & -0.3248 & 1.9211 & 0.068 \\
\hline$H(27 A)$ & $1 a$ & 0.4220 & -0.4874 & 1.9295 & 0.076 \\
\hline$H(27 B)$ & $1 a$ & 0.3323 & -0.4408 & 1.8557 & 0.076 \\
\hline$H(28 A)$ & $1 a$ & 0.8369 & -0.4441 & 1.9678 & 0.143 \\
\hline $\mathrm{H}(28 \mathrm{~B})$ & $1 a$ & 0.9038 & -0.5537 & 1.9301 & 0.143 \\
\hline $\mathrm{H}(28 \mathrm{C})$ & $1 a$ & 0.6927 & -0.5219 & 1.9683 & 0.143 \\
\hline$H(29 A)$ & $1 a$ & 0.8832 & -0.3799 & 1.7717 & 0.135 \\
\hline$H(29 B)$ & $1 a$ & 1.0158 & -0.4726 & 1.8076 & 0.135 \\
\hline $\mathrm{H}(29 \mathrm{C})$ & $1 a$ & 0.9631 & -0.3631 & 1.8461 & 0.135 \\
\hline$H(21 A)$ & $1 a$ & 0.7814 & -0.6200 & 1.7002 & 0.089 \\
\hline$H(21 B)$ & $1 a$ & 0.8886 & -0.5271 & 1.7101 & 0.089 \\
\hline$H(21 C)$ & $1 a$ & 0.7627 & -0.5218 & 1.6452 & 0.089 \\
\hline H(31) & $1 a$ & 0.0275 & -0.2605 & 1.2592 & 0.071 \\
\hline$H(34 A)$ & $1 a$ & 0.3076 & -0.3185 & 1.0303 & 0.103 \\
\hline$H(34 B)$ & $1 a$ & 0.0944 & -0.3098 & 1.0172 & 0.103 \\
\hline$H(35)$ & $1 a$ & 0.1715 & -0.452 & 1.1078 & 0.092 \\
\hline$H(37 A)$ & $1 a$ & 0.2719 & -0.4019 & 1.2215 & 0.103 \\
\hline $\mathrm{H}(37 \mathrm{~B})$ & $1 a$ & 0.3576 & -0.3151 & 1.1666 & 0.103 \\
\hline $\mathrm{H}(38 \mathrm{~A})$ & $1 a$ & -0.2053 & -0.4019 & 1.2609 & 0.159 \\
\hline
\end{tabular}

Table 2. Continued.

\begin{tabular}{|c|c|c|c|c|c|}
\hline Atom & Site & $x$ & $y$ & $z$ & $U_{\text {iso }}$ \\
\hline $\mathrm{H}(38 \mathrm{~B})$ & $1 a$ & 0.0101 & -0.4543 & 1.2536 & 0.159 \\
\hline $\mathrm{H}(38 \mathrm{C})$ & $1 a$ & -0.1223 & -0.4869 & 1.2001 & 0.159 \\
\hline$H(39 A)$ & $1 a$ & -0.1789 & -0.2510 & 1.0899 & 0.163 \\
\hline H(39B) & $1 a$ & -0.3146 & -0.2627 & 1.1651 & 0.163 \\
\hline$H(39 C)$ & $1 a$ & -0.2587 & -0.3558 & 1.1090 & 0.163 \\
\hline$H(31 A)$ & $1 a$ & -0.1312 & -0.0709 & 1.2290 & 0.099 \\
\hline$H(31 B)$ & $1 a$ & -0.2062 & -0.1088 & 1.1591 & 0.099 \\
\hline$H(3 I C)$ & $1 a$ & -0.0824 & -0.0195 & 1.1494 & 0.099 \\
\hline$H(41)$ & $1 a$ & 0.4804 & 0.0855 & 1.1966 & 0.065 \\
\hline$H(44 A)$ & $1 a$ & 0.1836 & 0.3586 & 1.0839 & 0.084 \\
\hline$H(44 B)$ & la & 0.3930 & 0.3718 & 1.0519 & 0.084 \\
\hline$H(45)$ & $1 a$ & 0.3586 & 0.3762 & 1.1880 & 0.078 \\
\hline$H(47 A)$ & $1 a$ & 0.1501 & 0.2210 & 1.1861 & 0.087 \\
\hline $\mathrm{H}(47 \mathrm{~B})$ & $1 a$ & 0.2624 & 0.2267 & 1.2562 & 0.087 \\
\hline$H(48 A)$ & $1 a$ & 0.7484 & 0.1807 & 1.2374 & 0.096 \\
\hline $\mathrm{H}(48 \mathrm{~B})$ & la & 0.5485 & 0.2345 & 1.2768 & 0.096 \\
\hline$H(48 C)$ & $1 a$ & 0.6931 & 0.3036 & 1.2364 & 0.096 \\
\hline$H(49 A)$ & $1 a$ & 0.7687 & 0.3097 & 1.1016 & 0.090 \\
\hline H(49B) & $1 a$ & 0.6728 & 0.2471 & 1.0497 & 0.090 \\
\hline$H(49 C)$ & $1 a$ & 0.8229 & 0.1869 & 1.0987 & 0.090 \\
\hline $\mathrm{H}(41 \mathrm{~A})$ & $1 a$ & 0.5637 & -0.0251 & 1.0748 & 0.082 \\
\hline$H(41 B)$ & $1 a$ & 0.6666 & 0.0662 & 1.0388 & 0.082 \\
\hline $\mathrm{H}(41 \mathrm{C})$ & $1 a$ & 0.5138 & 0.0276 & 0.9980 & 0.082 \\
\hline$H(51)$ & $1 a$ & -0.0657 & -0.0319 & 0.8670 & 0.071 \\
\hline$H(54 A)$ & $1 a$ & 0.3197 & 0.0904 & 0.6887 & 0.087 \\
\hline$H(54 B)$ & $1 a$ & 0.1270 & 0.1614 & 0.6743 & 0.087 \\
\hline H(55) & $1 a$ & 0.1029 & -0.0216 & 0.6629 & 0.081 \\
\hline$H(57 A)$ & $1 a$ & 0.1407 & -0.1320 & 0.7727 & 0.085 \\
\hline$H(57 B)$ & $1 a$ & 0.2855 & -0.0560 & 0.7863 & 0.085 \\
\hline$H(58 A)$ & $1 a$ & -0.3307 & -0.0470 & 0.7857 & 0.134 \\
\hline H(58B) & $1 a$ & -0.1443 & -0.1214 & 0.7521 & 0.134 \\
\hline$H(58 C)$ & $1 a$ & -0.2646 & -0.0463 & 0.6987 & 0.134 \\
\hline$H(59 A)$ & $1 a$ & -0.2588 & 0.1472 & 0.6948 & 0.138 \\
\hline $\mathrm{H}(59 \mathrm{~B})$ & $1 a$ & -0.1691 & 0.1936 & 0.7575 & 0.138 \\
\hline $\mathrm{H}(59 \mathrm{C})$ & $1 a$ & -0.3504 & 0.1411 & 0.7792 & 0.138 \\
\hline$H(51 A)$ & $1 a$ & -0.1021 & 0.1356 & 0.9500 & 0.124 \\
\hline $\mathrm{H}(51 \mathrm{~B})$ & $1 a$ & -0.1842 & 0.1904 & 0.8789 & 0.124 \\
\hline $\mathrm{H}(51 \mathrm{C})$ & la & -0.0234 & 0.2337 & 0.9117 & 0.124 \\
\hline
\end{tabular}

Table 3. Atomic coordinates and displacement parameters (in $\AA^{2}$ ).

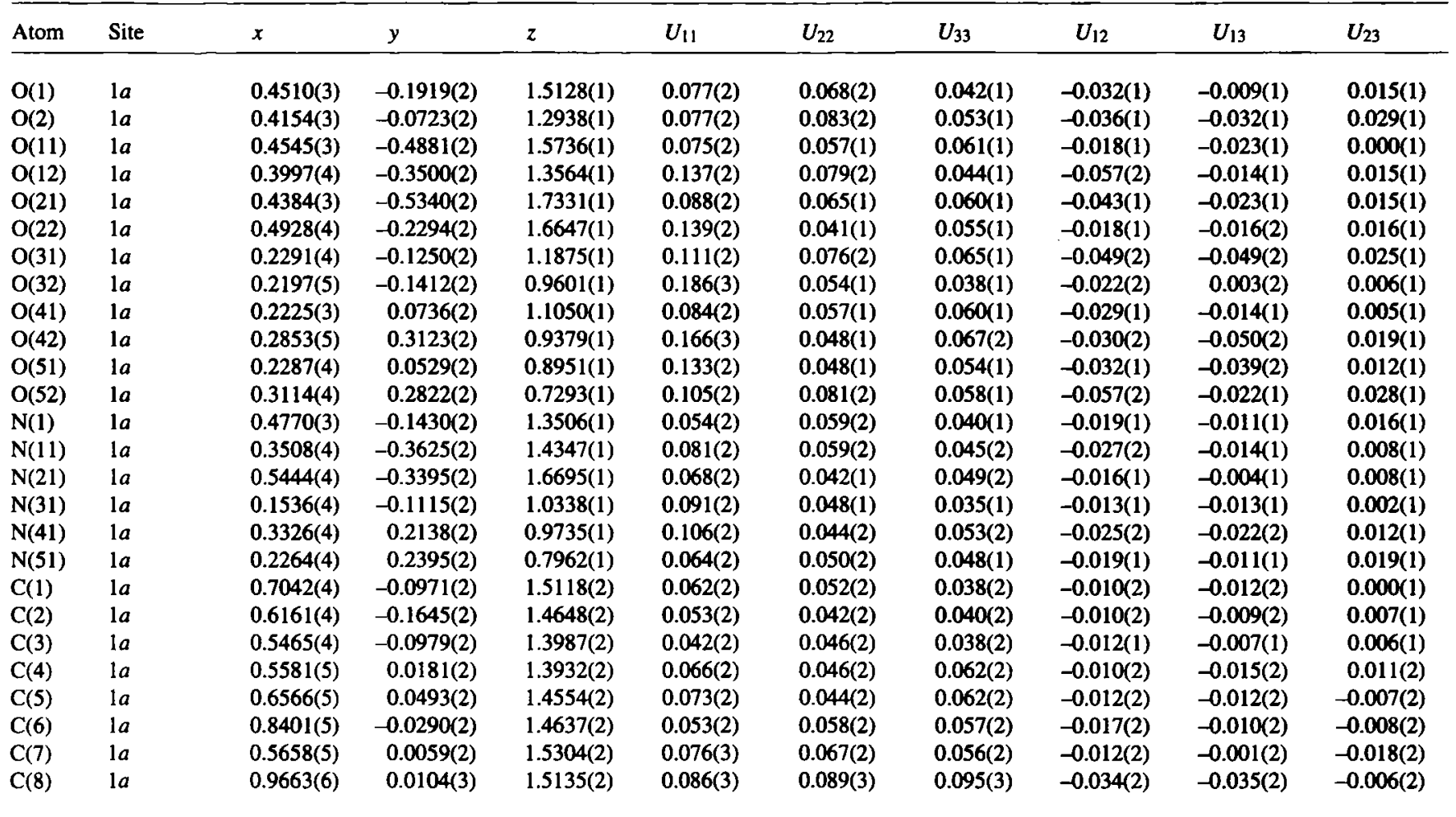


Table 3. Continued.

\begin{tabular}{|c|c|c|c|c|c|c|c|c|c|c|}
\hline Atom & Site & $x$ & $y$ & $z$ & $U_{11}$ & $U_{22}$ & $U_{33}$ & $U_{12}$ & $U_{13}$ & $U_{23}$ \\
\hline$C(9)$ & $1 a$ & $0.9660(5)$ & $-0.0698(3)$ & $1.3931(2)$ & $0.062(2)$ & $0.087(3)$ & $0.078(2)$ & $-0.024(2)$ & $-0.001(2)$ & $-0.008(2)$ \\
\hline$C(10)$ & $1 a$ & $0.7505(5)$ & $-0.2651(2)$ & $1.4424(2)$ & $0.077(2)$ & $0.048(2)$ & $0.071(2)$ & $-0.005(2)$ & $-0.027(2)$ & $-0.001(2)$ \\
\hline$C(11)$ & $1 a$ & $0.2333(4)$ & $-0.5901(2)$ & $1.5478(2)$ & $0.063(2)$ & $0.045(2)$ & $0.048(2)$ & $-0.012(2)$ & $-0.007(2)$ & $0.006(1)$ \\
\hline$C(12)$ & $1 a$ & $0.2847(4)$ & $-0.4804(2)$ & $1.5379(2)$ & $0.054(2)$ & $0.044(2)$ & $0.047(2)$ & $-0.011(2)$ & $-0.012(2)$ & $0.004(1)$ \\
\hline$C(13)$ & $1 a$ & $0.3374(5)$ & $-0.4579(2)$ & $1.4543(2)$ & $0.066(2)$ & $0.049(2)$ & $0.047(2)$ & $-0.021(2)$ & $-0.010(2)$ & $0.001(2)$ \\
\hline$C(14)$ & $1 a$ & $0.3696(6)$ & $-0.5459(2)$ & $1.3993(2)$ & $0.089(3)$ & $0.063(2)$ & $0.060(2)$ & $-0.024(2)$ & $-0.002(2)$ & $-0.008(2)$ \\
\hline$C(16)$ & $1 a$ & $0.1139(5)$ & $-0.6171(2)$ & $1.4873(2)$ & $0.065(2)$ & $0.041(2)$ & $0.063(2)$ & $-0.015(2)$ & $-0.012(2)$ & $0.003(2)$ \\
\hline$C(17)$ & $1 a$ & $0.3996(5)$ & $-0.6694(2)$ & $1.5094(2)$ & $0.069(2)$ & $0.044(2)$ & $0.087(3)$ & $-0.001(2)$ & $-0.011(2)$ & $-0.003(2)$ \\
\hline$C(18)$ & $1 a$ & $0.0261(6)$ & $-0.7146(3)$ & $1.5114(2)$ & $0.109(3)$ & $0.061(2)$ & $0.087(3)$ & $-0.039(2)$ & $-0.017(2)$ & $-0.002(2)$ \\
\hline$C(19)$ & $1 a$ & $-0.0385(6)$ & $-0.5356(3)$ & $1.4569(2)$ & $0.080(3)$ & $0.064(2)$ & $0.088(3)$ & $-0.018(2)$ & $-0.029(2)$ & $0.001(2)$ \\
\hline$C(110)$ & $1 a$ & $0.1314(5)$ & $-0.3966(2)$ & $1.5767(2)$ & $0.079(3)$ & $0.047(2)$ & $0.060(2)$ & $-0.012(2)$ & $-0.004(2)$ & $-0.002(2)$ \\
\hline $\mathrm{C}(21)$ & $1 a$ & $0.6190(5)$ & $-0.5168(2)$ & $1.8302(2)$ & $0.074(2)$ & $0.043(2)$ & $0.050(2)$ & $-0.005(2)$ & $-0.015(2)$ & $0.008(1)$ \\
\hline$C(22)$ & $1 a$ & $0.6033(4)$ & $-0.4922(2)$ & $1.7481(2)$ & $0.047(2)$ & $0.041(2)$ & $0.048(2)$ & $-0.012(1)$ & $-0.004(2)$ & $0.003(1)$ \\
\hline$C(25)$ & $1 a$ & $0.5601(5)$ & $-0.3662(2)$ & $1.8755(2)$ & $0.073(2)$ & $0.057(2)$ & $0.040(2)$ & $-0.016(2)$ & $-0.001(2)$ & $-0.000(1)$ \\
\hline$C(26)$ & $1 a$ & $0.7424(5)$ & $-0.4474(3)$ & $1.8644(2)$ & $0.060(2)$ & $0.072(2)$ & $0.065(2)$ & $-0.003(2)$ & $-0.022(2)$ & $-0.006(2)$ \\
\hline$C(27)$ & $1 a$ & $0.4471(5)$ & $-0.4567(3)$ & $1.8795(2)$ & $0.067(2)$ & $0.079(2)$ & $0.043(2)$ & $-0.022(2)$ & $0.001(2)$ & $0.013(2)$ \\
\hline$C(28)$ & $1 a$ & $0.7993(8)$ & $-0.4964(4)$ & $1.9398(2)$ & $0.150(5)$ & $0.109(3)$ & $0.103(3)$ & $0.009(3)$ & $-0.074(3)$ & $-0.007(3)$ \\
\hline$C(29)$ & $1 a$ & $0.9175(6)$ & $-0.4125(4)$ & $1.8181(3)$ & $0.055(3)$ & $0.166(5)$ & $0.127(4)$ & $-0.031(3)$ & $-0.015(3)$ & $-0.047(3)$ \\
\hline$C(210)$ & $1 a$ & $0.7747(5)$ & $-0.5451(3)$ & $1.6962(2)$ & $0.085(3)$ & $0.060(2)$ & $0.070(2)$ & $0.003(2)$ & $-0.002(2)$ & $-0.012(2)$ \\
\hline$C(31)$ & $1 a$ & $0.0649(5)$ & $-0.2685(2)$ & $1.2049(2)$ & $0.075(2)$ & $0.061(2)$ & $0.044(2)$ & $-0.022(2)$ & $-0.003(2)$ & $0.008(2)$ \\
\hline$C(32)$ & $1 a$ & $0.0778(4)$ & $-0.1657(2)$ & $1.1614(2)$ & $0.056(2)$ & $0.051(2)$ & $0.039(2)$ & $-0.011(2)$ & $-0.012(2)$ & $0.001(1)$ \\
\hline$C(33)$ & $1 a$ & $0.1365(4)$ & $-0.1894(2)$ & $1.0785(2)$ & $0.056(2)$ & $0.041(2)$ & $0.040(2)$ & $-0.003(2)$ & $-0.007(1)$ & $0.002(1)$ \\
\hline$C(34)$ & $1 a$ & $0.1765(7)$ & $-0.3016(2)$ & $1.0541(2)$ & $0.138(4)$ & $0.043(2)$ & $0.061(2)$ & $-0.003(2)$ & $0.023(2)$ & $-0.002(2)$ \\
\hline$C(35)$ & $1 a$ & $0.1439(6)$ & $-0.3774(2)$ & $1.1201(2)$ & $0.118(3)$ & $0.036(2)$ & $0.066(2)$ & $-0.012(2)$ & $0.014(2)$ & $0.001(2)$ \\
\hline$C(36)$ & $1 a$ & $-0.0439(6)$ & $-0.3421(3)$ & $1.1691(2)$ & $0.082(3)$ & $0.076(2)$ & $0.067(2)$ & $-0.039(2)$ & $0.002(2)$ & $-0.003(2)$ \\
\hline$C(37)$ & $1 a$ & $0.2460(6)$ & $-0.3467(3)$ & $1.1839(2)$ & $0.093(3)$ & $0.058(2)$ & $0.104(3)$ & $-0.007(2)$ & $-0.019(3)$ & $0.037(2)$ \\
\hline$C(38)$ & $1 a$ & $-0.0952(8)$ & $-0.4296(4)$ & $1.2264(3)$ & $0.182(5)$ & $0.114(4)$ & $0.107(3)$ & $-0.089(4)$ & $0.041(4)$ & $0.000(3)$ \\
\hline$C(39)$ & $1 a$ & $-0.2146(7)$ & $-0.2989(4)$ & $1.1297(3)$ & $0.100(4)$ & $0.148(5)$ & $0.181(5)$ & $-0.058(4)$ & $-0.034(4)$ & $-0.044(4)$ \\
\hline$C(310)$ & $1 a$ & $-0.1021(6)$ & $-0.0837(3)$ & $1.1761(2)$ & $0.093(3)$ & $0.071(2)$ & $0.073(2)$ & $0.006(2)$ & $-0.002(2)$ & $-0.010(2)$ \\
\hline$C(43)$ & $1 a$ & $0.3457(5)$ & $0.2224(2)$ & $1.0432(2)$ & $0.072(2)$ & $0.041(2)$ & $0.048(2)$ & $-0.015(2)$ & $-0.012(2)$ & $-0.000(1)$ \\
\hline$C(44)$ & $1 a$ & $0.3163(6)$ & $0.3257(2)$ & $1.0813(2)$ & $0.096(3)$ & $0.042(2)$ & $0.073(2)$ & $-0.005(2)$ & $-0.024(2)$ & $-0.008(2)$ \\
\hline$C(45)$ & $1 a$ & $0.3712(5)$ & $0.3113(2)$ & $1.1604(2)$ & $0.087(3)$ & $0.052(2)$ & $0.057(2)$ & $-0.009(2)$ & $-0.010(2)$ & $-0.015(2)$ \\
\hline$C(46)$ & $1 a$ & $0.5621(5)$ & $0.2354(2)$ & $1.1612(2)$ & $0.072(2)$ & $0.055(2)$ & $0.049(2)$ & $-0.023(2)$ & $-0.006(2)$ & $-0.004(2)$ \\
\hline$C(47)$ & $1 a$ & $0.2717(6)$ & $0.2255(3)$ & $1.2019(2)$ & $0.079(3)$ & $0.089(3)$ & $0.053(2)$ & $-0.028(2)$ & $0.004(2)$ & $-0.015(2)$ \\
\hline$C(48)$ & $1 a$ & $0.6459(6)$ & $0.2389(3)$ & $1.2349(2)$ & $0.097(3)$ & $0.080(2)$ & $0.068(2)$ & $-0.025(2)$ & $-0.019(2)$ & $-0.003(2)$ \\
\hline$C(49)$ & $1 a$ & $0.7214(5)$ & $0.2457(3)$ & $1.0968(2)$ & $0.082(3)$ & $0.072(2)$ & $0.074(2)$ & $-0.029(2)$ & $-0.001(2)$ & $-0.004(2)$ \\
\hline$C(410)$ & $1 a$ & $0.5487(5)$ & $0.0397(2)$ & $1.0460(2)$ & $0.093(3)$ & $0.045(2)$ & $0.067(2)$ & $-0.006(2)$ & $-0.014(2)$ & $-0.002(2)$ \\
\hline$C(51)$ & $1 a$ & $0.0001(5)$ & $0.0073(2)$ & $0.8265(2)$ & $0.071(2)$ & $0.047(2)$ & $0.062(2)$ & $-0.025(2)$ & $-0.003(2)$ & $0.009(2)$ \\
\hline$C(52)$ & $1 a$ & $0.0778(5)$ & $0.0998(2)$ & $0.8527(2)$ & $0.069(2)$ & $0.043(2)$ & $0.046(2)$ & $-0.017(2)$ & $-0.001(2)$ & $0.002(1)$ \\
\hline$C(53)$ & $1 a$ & $0.1673(4)$ & $0.1554(2)$ & $0.7842(2)$ & $0.048(2)$ & $0.042(2)$ & $0.042(2)$ & $-0.011(1)$ & $-0.011(1)$ & $0.009(1)$ \\
\hline$C(54)$ & $1 a$ & $0.1858(6)$ & $0.1091(3)$ & $0.7083(2)$ & $0.086(3)$ & $0.090(3)$ & $0.050(2)$ & $-0.038(2)$ & $-0.014(2)$ & $0.006(2)$ \\
\hline$C(55)$ & $1 a$ & $0.0929(5)$ & $0.0126(3)$ & $0.7111(2)$ & $0.083(3)$ & $0.069(2)$ & $0.060(2)$ & $-0.027(2)$ & $-0.021(2)$ & $-0.006(2)$ \\
\hline$C(56)$ & $1 a$ & $-0.1059(5)$ & $0.0325(3)$ & $0.7558(2)$ & $0.058(2)$ & $0.053(2)$ & $0.102(3)$ & $-0.020(2)$ & $-0.029(2)$ & $0.009(2)$ \\
\hline$C(57)$ & $1 a$ & $0.1581(5)$ & $-0.0598(2)$ & $0.7761(2)$ & $0.086(3)$ & $0.048(2)$ & $0.083(2)$ & $-0.009(2)$ & $-0.035(2)$ & $-0.007(2)$ \\
\hline$C(58)$ & $1 a$ & $-0.2222(6)$ & $-0.0536(3)$ & $0.7473(3)$ & $0.096(3)$ & $0.093(3)$ & $0.167(5)$ & $-0.051(3)$ & $-0.053(3)$ & $0.013(3)$ \\
\hline$C(59)$ & $1 a$ & $-0.2328(6)$ & $0.1384(3)$ & $0.7459(3)$ & $0.073(3)$ & $0.081(3)$ & $0.198(5)$ & $-0.004(2)$ & $-0.059(3)$ & $0.011(3)$ \\
\hline$C(510)$ & $1 a$ & $-0.0716(6)$ & $0.1714(3)$ & $0.9029(2)$ & $0.117(4)$ & $0.084(3)$ & $0.103(3)$ & $-0.043(3)$ & $0.052(3)$ & $-0.036(2)$ \\
\hline
\end{tabular}

Acknowledgment. We acknowledge financial support by the Education Commission of Zhejiang Province (No. 20010129).

\section{References}

1. Yan, J. Z.; Lu, Y. P.; Sun, N.; He, L.; Jin, Z. M.: Crystal Structure of 4methylpyridinium 2,4-dinitrophenolate. J. Chem. Crystallogr. 31 (2001) 293-296.

2. Jin Z. M.; Pan Y. J.; Hu M. L.; Zhou J. W.: The crystal structure of an anomalous and tautomeric salt: 2:1 complex of $(E)-2(1 H)$-6-methylpyridiniminium hydrogen maleate with maleic acid. J. Mol. Struct. 609 (2002) 83-87.

3. Usman, A.; Chantrapromma, S.; Fun, H. K.; Poh, B. L.; Karalai, C.: A 1:1 adduct hexamehtylenetetramine and 4-hydroxy-3-methoxy-benzaldehyde. Acta Crystallogr. C58 (2002) o48-050.
4. Sheldrick, G. M.: SHELXTL-plus. Siemens Analytical X-ray Instruments, Inc. 6300 Enterprise Lane, Madison, WI 53719-1173, USA 1990.

5. Sheldrick, G. M.: SHELXL-93. Program for Crystal Structure Refinement. University of Göttingen, Göttingen, Germany 1993.

6. Farrugia, L. J.: ORTEP3 for Windows. University of Glasgow, Glasgow G12 8QQ, Scotland 1997. 